\title{
Tuberculosis cutánea: reporte de dos casos y revisión de la literatura
}

\author{
Marcela Concha R., Félix Fich S., Ricardo Rabagliati B., Cristian Pinto S., \\ Rocío Rubio L., Óscar Navea D. y Sergio González B.
}

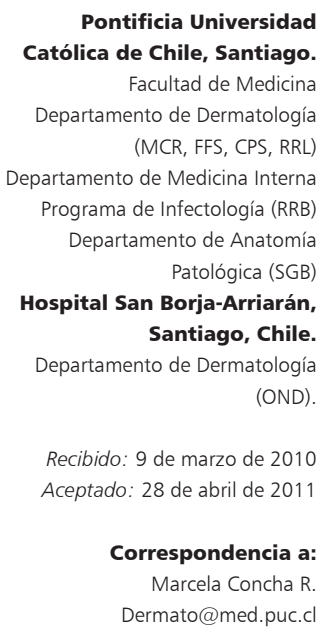

Pontificia Universidad Católica de Chile, Santiago. Facultad de Medicina Departamento de Dermatología (MCR, FFS, CPS, RRL) Departamento de Medicina Interna Programa de Infectología (RRB) Departamento de Anatomía Patológica (SGB) Hospital San Borja-Arriarán, Santiago, Chile. Departamento de Dermatología (OND).

Recibido: 9 de marzo de 2010 Aceptado: 28 de abril de 2011

Correspondencia a: Marcela Concha R. Dermato@med.puc.c

\section{Caso 1}

$\mathrm{P}$ aciente de 56 años, sexo femenino, con antecedente de dermatomiositis diagnosticada un año previo a la consulta, en tratamiento con hidroxicloroquina (Plaquinol ${ }^{\circledR}$ ) $200 \mathrm{mg}$, metotrexato $10 \mathrm{mg}$ y prednisona 30 mg diarios. Fue hospitalizada por cuadro de 10 días de evolución, con fiebre, compromiso del estado general y pérdida de peso, encontrándose crepitaciones bilaterales al examen pulmonar. La TAC de tórax mostró un infiltrado nodular bilateral, extenso, con cavitaciones biapicales. La baciloscopia obtenida por lavado bronquio-alveolar (LBA) resultó positiva y la reacción de polimerasa en cadena (RPC) positiva para Mycobacterium tuberculosis. El resto del estudio de LBA fue negativo (cultivo corriente, hongos, Nocardia spp, inmunofluorescencia, RPC para Pneumocystis jiroveci, panel viral respiratorio, cultivo de citomegalovirus, galactomanano). El Departamento de Dermatología fue consultado por úlceras en los pulgares aparecidas cuatro semanas previas al ingreso. Al examen físico se observó una úlcera de bordes bien delimitados con fondo sucio y bordes necróticos, no dolorosa, ubicada en el pulpejo del dedo pulgar izquierdo (Figuras 1A y B). Se le realizó biopsia que mostró una necrosis reciente extensa, sin evidencia de vasculitis ni elementos histopatológicos sugerentes de TBC, con inmunofluorescencia directa para C3, IgA, IgG, IgM y fibrina negativos. En el cultivo corriente crecieron $S$. aureus y Candida parapsilosis. La tinción de Ziehl Neelsen en tejido fue positiva. Se inició tratamiento con isoniacida, (HIN) rifampicina (RFP), pirazinamida (PZ) y etambutol, además de ceftriaxona y fluconazol. El cultivo acelerado de Mycobacterium spp de la úlcera del pulgar fue negativo a los 60 días; sin embargo, el cultivo obtenido en LBA resultó positivo a los 30 días. La paciente fue trasladada al Hospital del Tórax para continuar su tratamiento.

Dado que se trataba de una paciente inmunosuprimida que cursaba con un cuadro de tuberculosis (TBC) pulmonar, la úlcera del pulgar pudo corresponder a un absceso frío.

\section{Caso 2}

Paciente de 63 años, sexo femenino, con antecedentes de TBC pulmonar tratada a los nueve años de edad. Consultó por un cuadro de dos meses de evolución caracterizado por aumento de volumen, de consistencia dura, bien delimitado, ubicado en la mejilla izquierda, adyacente al polo inferior de la parótida, asociado a eritema perilesional (Figura 2A). Al examen físico, se identificó en la zona pre-auricular una herida lineal de bordes irregulares con exudado seropurulento (en un área donde se había realizado un drenaje previo en otro centro) (Figura 2B); además, en la zona frontal izquierda se observaba una placa café negruzca de superficie verrucosa, bien delimitada, friable al tacto (Figura 2C) y en la cara anterior de ambas piernas, la presencia de nódulos eritematosos, dolorosos, simétricos. La paciente no relataba síntomas sistémicos como fiebre, sudoración o baja de peso. Los exámenes de laboratorio general demostraron una VHS de $58 \mathrm{~mm} / \mathrm{h}$ y PCR de $22 \mathrm{mg} / \mathrm{L}$, examen de orina normal, ELISA para VIH negativo y radiografía de tórax sin evidencias de secuelas apicales. 
Se solicitó una ecografía cervical que fue informada como: "lesión de tipo inflamatoria, flegmonosa, con probable área de abscedación en situación infraparotídea izquierda”.

Se realizaron biopsias cutáneas que concluyeron: "proceso granulomatoso necrosante y supurativo, neutrofílico, en la lesión frontal.” (Figura 2D). “Dermatitis perivascular superficial y profunda de la región supra-parotídea y paniculitis septal, compatible con eritema nodoso en las lesiones de las extremidades inferiores".

La tinción de Ziehl Neelsen y el cultivo para micobacterias fue negativo en el exudado seropurulento preauricular y en la biopsia de la lesión frontal. También
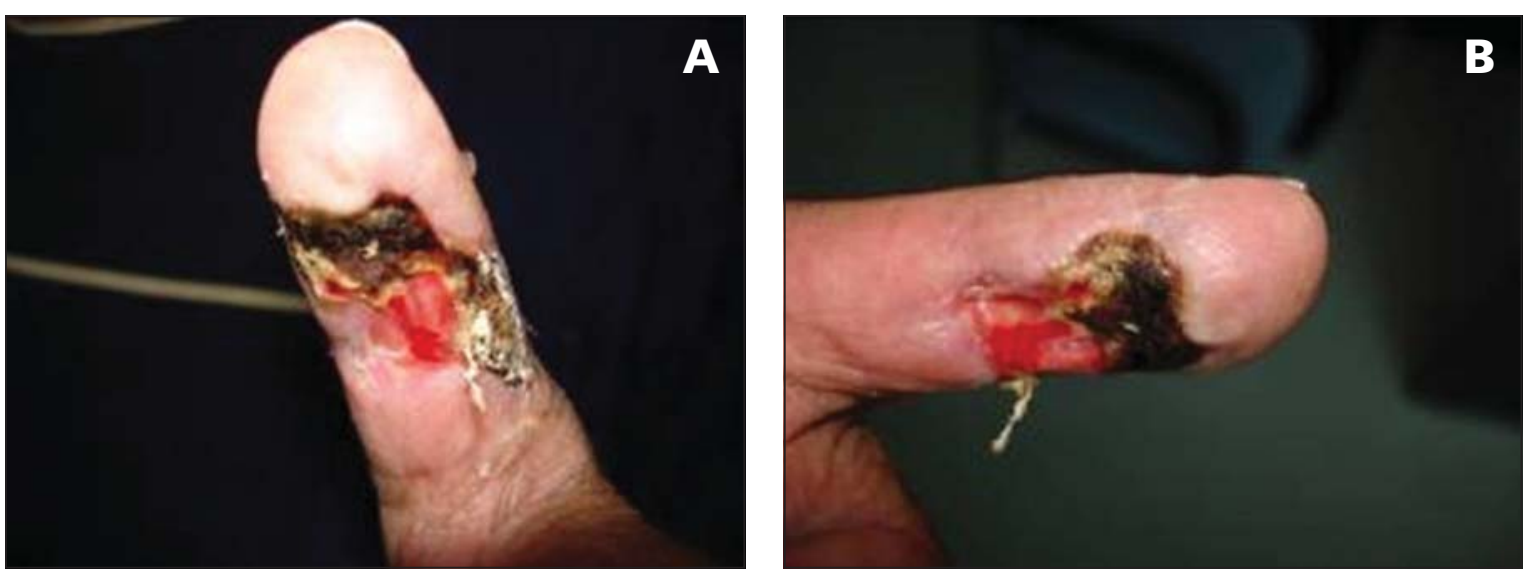

Figura 1. Aspecto clínico de lesiones en pulpejos.
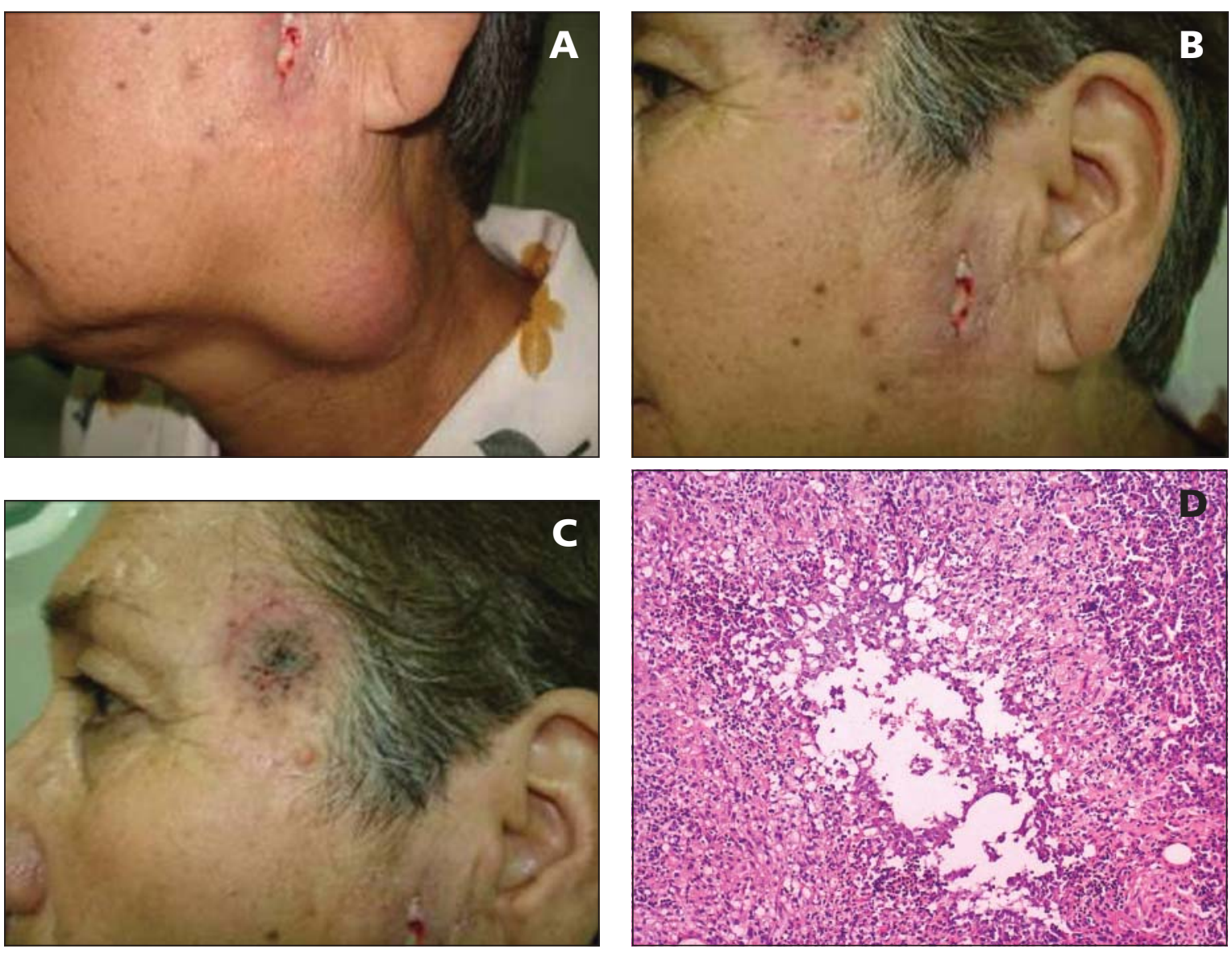

Figura 2. $A, B, C$. Aspecto clínico de las lesiones en la mejilla izquierda, preauricular y frontal, respectivamente. D. Aspecto histológico en biopsia de la lesión frontal (granuloma). 


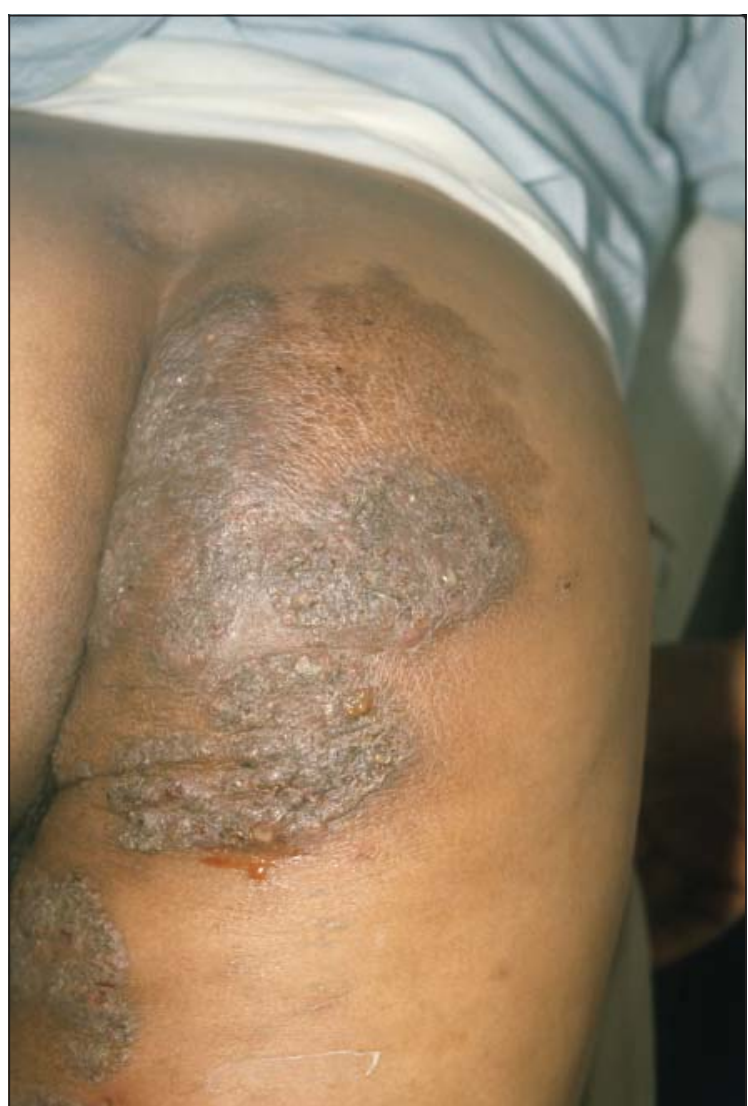

Figura 3.

la RPC para $M$. tuberculosis fue negativa en una muestra de tejido que evidenció mala preservación del ADN.

Debido al contexto clínico de la paciente y el resultado de la biopsia, se decidió iniciar tratamiento anti TBC con HIN, RFP y PZ, en dosis habituales para la fase diaria de los primeros dos meses, y rifampicina e isoniazida para la fase bisemanal de los siguientes cuatro meses. Luego del primer mes de terapia, la paciente notó una mejoría progresiva, con regresión de los signos inflamatorios y de la supuración en las lesiones faciales y desaparición de las lesiones de eritema nodoso. Luego de ocho semanas hubo normalización de la PCR y la VHS. Actualmente, la paciente completó el tratamiento antituberculoso, observándose regresión clínica y radiológica completa de las lesiones.

En conjunto, la clínica y la buena respuesta a terapia antituberculosa, nos permite plantear que esta paciente presentaba un escrofuloderma.

\section{Revisión de tuberculosis cutánea}

La TBC ha sido un problema de salud pública desde tiempos muy antiguos; actualmente ha recobrado im-

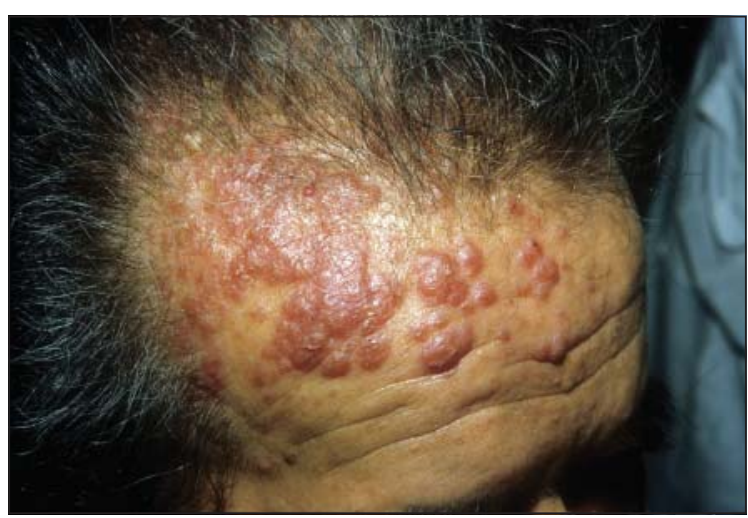

Figura 4.

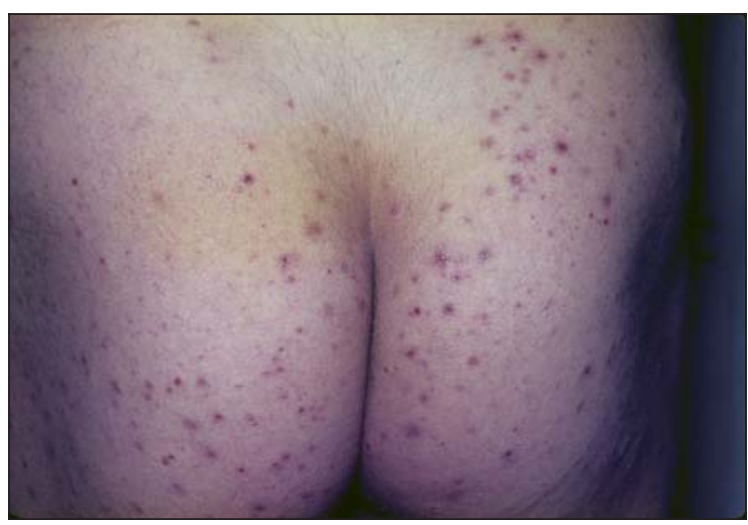

Figura 5.

portancia debido a la pandemia del VIH, al creciente número de personas que se encuentra en tratamiento inmunosupresor por diversas causas, las inmigraciones y al surgimiento de cepas multiresistentes ${ }^{1}$.

Se estima que en el año 2006 la prevalencia de TBC en Chile fue de 13,3/100.000 habitantes². Chile tendría la menor prevalencia de TBC entre los países de Sudamérica; en el extremo opuesto se encuentra Bolivia, con una estimación de 198 casos/100.000 habitantes para el mismo año³

Las puertas de entrada de la infección son, generalmente, la vía pulmonar e intestinal, siendo la TBC cutánea una manifestación de compromiso sistémico, aunque existe el compromiso primario de piel $^{4}$.

Como agentes etiológicos destaca Mycobacterium tuberculosis, aunque Mycobacterium bovis y el bacilo de Calmette-Guérin, utilizado en la vacunación anti-TBC, también es capaz de producir lesiones cutáneas ${ }^{5,6}$.

La TBC cutánea es una pequeña proporción de todos los casos de TBC, entre 1 y $2 \%$, pero en países con alta prevalencia de la enfermedad, cobra importancia el número de casos. Escrofuloderma y lupus vulgar son las dos formas más comunes ${ }^{7,8}$. La asociación entre TBC cutánea y visceral se da en aproximadamente $28 \%$ de los $\operatorname{casos}^{9}$. 


\section{Diagnóstico}

El diagnóstico clínico puede ser difícil debido a la gran variabilidad morfológica de las lesiones. Factores que modifican la presentación clínica de la enfermedad son la patogenicidad del microorganismo, su patrón de resistencia antimicrobiana, la puerta de entrada de la infección y el estado inmunológico del paciente, además de factores locales de la piel ${ }^{4}$.

El diagnóstico se basa en la historia clínica, el examen físico, la histopatología y pruebas de laboratorio. Dentro de estas últimas, se encuentran la tinción Ziehl Neelsen y la RPC; sin embargo, el cultivo continúa siendo el método más confiable para el diagnóstico de micobacterias vivas y la realización, en forma consecuente, del estudio de sensibilidad a antimicrobianos ${ }^{4}$. De ayuda en el diagnóstico puede ser el PPD. Recientemente aprobado por la FDA y de ayuda diagnóstica para los casos de TBC latente o activa, es la medición de $\gamma$ interferón (INF) en sangre total, luego de la exposición a antígenos de $M$. tuberculosis. Si el paciente está infectado con M. tuberculosis, sus linfocitos producen mayor cantidad de $\gamma$ INF frente a la exposición a estos antígenos. Estos antígenos no se encuentran en el bacilo de Calmette-Guérin ni en otras micobacterias no tuberculosas (excepto $M$. kansasii, $M$. szulgai y M. marinum), por lo que esta prueba sería más específica $^{10,11}$.

Es recomendable realizar el test de ELISA para VIH a los pacientes, ya que la TBC es más frecuente en este grupo de pacientes ${ }^{12}$.

\section{Clasificación}

Una forma de clasificar los diferentes tipos de TBC cutánea es de acuerdo al tipo de diseminación (Tabla 1). Los casos de infección exógena ocurren después de inoculación directa del microorganismo en la piel, dentro de los que se incluyen el chancro tuberculoso, tuberculosis verrucosa cutis y algunos casos de lupus vulgar. La infección endógena ocurre en individuos previamente infectados, con diseminación del organismo hacia la piel por contigüidad, vía hematógena o linfática; aquí podemos nombrar la mayoría de los casos de lupus vulgar, escrofuloderma, TBC miliar, orificial y abscesos tuberculosos ${ }^{1}$.

Además, es posible realizar algunas distinciones de acuerdo a la carga bacteriana de las lesiones. En aquellas que se encuentra gran cantidad de bacilos ácido-alcohol resistente a la observación directa o gran cantidad de micobacterias en el cultivo, se denominarán multibacilares; en el caso contrario paucibacilares ${ }^{8}$. Las presentaciones pauci-inmunes generalmente presentan un test de PPD positivo, lo cual ayuda en el diagnóstico.
Chancro tuberculoso. Inoculación primaria de TBC cutánea (en un individuo que no se encuentra sensibilizado), ocurre frecuentemente en trabajadores de la salud o en laboratorios, que se infectan con material contaminado, o en niños que no han sido vacunados y tienen contacto con personas con TBC pulmonar ${ }^{1,8}$. También se ha descrito en heridas operatorias, tatuajes ${ }^{13}$, piercing, $y$ luego de respiración artificial boca a boca ${ }^{6}$. En el sitio de inoculación hay discontinuidad de la barrera cutánea (abrasiones, heridas, forúnculos) ${ }^{14}$.

La base de la úlcera es generalmente gruesa, granular, con tendencia al sangrado, de color rojo oscuro y sus bordes son indurados e irregulares ${ }^{15}$. Es indolora ${ }^{16}$.

El test de PPD es negativo inicialmente, pero se torna positivo durante el curso de la enfermedad ${ }^{1}$.

La histología temprana muestra una reacción inflamatoria aguda, abundantes neutrófilos, áreas de necrosis y bacilos tuberculosos. Luego de tres a seis semanas, la lesión toma aspecto granulomatoso, con aumento de células gigantes y epiteloídeas y disminución de bacilos ${ }^{1,6}$.

Tuberculosis verrucosa cutis. Usualmente comienza como una pápula o nódulo hiperqueratósico, verrucoso e indurado; progresa lentamente, de manera serpiginosa, resultando una placa roja-café, verrucosa, solitaria, indolora, de bordes irregulares, cuya superficie es atravesada por hendiduras y fisuras. La placa es generalmente firme, aunque ocasionalmente puede tener un centro suave y exudar material purulento o queratósico. Las lesiones son crónicas y progresan en forma centrifuga con curación

Tabla 1. Clasificación de la tuberculosis cutánea. Adaptado de Barbagallo et al ${ }^{1}$

\begin{tabular}{|c|c|c|}
\hline \multicolumn{3}{|l|}{ Infección exógena } \\
\hline Enfermedad & Ruta de Infección & Carga bacteriana \\
\hline Chancro tuberculoso & Inoculación directa & + \\
\hline Tuberculosis verrucosa cutis & Inoculación directa & \pm \\
\hline Lupus vulgar (algunos casos) & Inoculación directa, vacuna BCG & \pm \\
\hline \multicolumn{3}{|l|}{ Infección endógena } \\
\hline Enfermedad & Ruta de Infección & Carga bacteriana \\
\hline Lupus vulgar (mayoría de los casos) & Hematógena, linfática, contigüidad & \pm \\
\hline Escrofuloderma & Contigüidad & + \\
\hline Tuberculosis miliar & Hematógena & + \\
\hline Tuberculosis orificial & Autoinoculación & + \\
\hline Absceso tuberculoso & Hematógena & + \\
\hline Tuberculide papulonecrótico & Hematógena & - \\
\hline
\end{tabular}


central y se ubican generalmente en las extremidades ${ }^{17}$. (Figura 3)

Se debe a la re-inoculación de micobacterias en un individuo previamente expuesto, por lo que la vacunación no protege contra esta forma de $\mathrm{TBC}^{8}$. Generalmente se encuentra adenopatías regionales sólo en caso de sobreinfección secundaria ${ }^{1}$.

La inoculación de micobacterias ocurre en la piel con heridas; es frecuente en profesionales de la salud en contacto con pacientes con TBC o material de autopsia ${ }^{1}$, así como en veterinarios, empleados de mataderos, que están en contacto con animales infectados ${ }^{18}$.

La visualización de micobacterias es muy difícil (forma pauci-inmune $)^{8}$ y el cultivo es generalmente negativo ${ }^{1}$.

Lupus vulgar. Lesiones que pueden resultar de diseminación hematógena o re-inoculación; las primeras se ubican generalmente en la cara y las segundas en las extremidades ${ }^{8}$. Corresponde a una placa café rojiza, de lento crecimiento con borde verrucoso levemente solevantado y atrofia central. La placa está formada por micropápulas coalescentes o lupomas y tiene consistencia suave $^{8}$; al crecer hacia la periferia deja un centro atrófico ${ }^{1}$. Hay que considerar que también existen formas ulceradas, vegetantes e hipertróficas ${ }^{4,7}$.(Figura 4).

La histología muestra múltiples granulomas con ocasionales áreas de necrosis caseosa, particularmente en la dermis superior; se observan escasos bacilos o son ausentes ${ }^{1,8}$. Se pueden observar cambios secundarios de la piel como atrofia, ulceración o acantosis ${ }^{14}$.

El rendimiento del cultivo es pobre y el test de tuberculina es positivo; en caso contrario hay que buscar compromiso visceral que cause menor reactividad ${ }^{1}$.

Escrofuloderma. Causada por la propagación por contigüidad de la infección desde una estructura subyacente, como un linfonodo o hueso. Se forma un absceso que luego fistuliza, se puede observar induración del área circundante, resultando una úlcera rodeada de tejido queloídeo ${ }^{8}$. Los lugares más frecuentemente afectados son el cuello, axilas, pared torácica y la región inguinal ${ }^{8}$. Clínicamente se observa un nódulo rojo-café sobre un nódulo afectado, que se indura y luego se rompe, drenando material seroso, purulento o caseoso ${ }^{1}$.

El diagnóstico se realiza fácilmente por cultivo, biopsia u observación de bacilos mediante la tinción de Ziehl Neelsen ${ }^{1}$.

Tuberculosis miliar. Se observa en el contexto de una TBC diseminada, generalmente en niños y jóvenes; actualmente han aumentado los casos en adultos, principalmente en aquellos infectados con $\mathrm{VIH}^{1}$.

Se ha descrito un amplio espectro de lesiones, desde eritema a úlceras ${ }^{19}$. Las más comunes corresponden a máculas o pápulas eritematosas, pequeñas, que se rompen o secan, dando paso a costras que al ser retiradas revelan umbilicación. Se ubican, con mayor frecuencia, en el tron$\mathrm{Co}^{8,14}$. En algunas ocasiones, sin embargo, los hallazgos son indistinguibles de una celulitis o erisipela clásica ${ }^{19}$.

Tuberculosis periorificial. No es una presentación muy frecuente, ocurre en el contexto de la TBC avanzada intestinal, pulmonar o genitourinaria. Las lesiones son introducidas en la piel periorificial y mucosas por autoinoculación desde dichos sitios ${ }^{1,8}$. Si el compromiso es oral, con frecuencia el foco primario se encuentra en la vía aérea superior o los pulmones; si es perineal, en el tracto digestivo o genitourinario ${ }^{8}$.

Las lesiones se localizan en la mucosa oral, perianal, alrededor de la vulva o pene y corresponden generalmente a úlceras dolorosas sobre una base pseudomembranosa fibrinosa, aunque en ausencia de úlcera puede haber una placa o tejido hipertrófico ${ }^{8}$.

Abscesos fríos (Gomas). Son producto de la diseminación metastásica, vía hematógena, de micobacterias latentes, formando abscesos en las extremidades o el tronco generalmente, sin compromiso de los tejidos profundos ${ }^{8}$. Pueden romperse hacia el exterior, siendo clínicamente indistinguible del escrofuloderma ${ }^{1}$.

Tuberculides. Este término se introdujo en 1896 para designar aquellas dermatosis que se caracterizan por presentarse en individuos con historia de TBC activa, con histología tuberculoidea, reacción tuberculínica intensamente positiva, pero en cuyos cultivos no se lograban identificar micobacterias. Posteriormente se agrega la característica buena respuesta a terapia anti $\mathrm{TBC}^{20}$. Su etiopatogenia no es clara, antiguamente se pensaba que correspondían a la reacción inmune a la presencia de micobacterias en el organismo, pero actualmente se ha comprobado que también en la lesión se pueden encontrar bacilos de Koch mediante técnicas como la RPC. Corresponden al extremo paucibacilar de la TBC cutánea ${ }^{8}$.

Tuberculides papulo-necróticas. Son múltiples pápulas rojas oscuras, que pueden volverse costrosas o ulceradas, ubicadas generalmente en las superficies extensoras de las extremidades. Puede dejar cicatrices atróficas y pigmentadas. (Figura 5). La presencia de lesiones activas adyacentes a cicatrices son de ayuda diagnóstica para tuberculides papulo-necróticas ${ }^{1,4,8}$. Frecuentemente aparecen en personas con enfermedad activa no cutánea ${ }^{8}$.

Eritema indurado de Bazin. Se presenta como nódulos violáceos indoloros, con tendencia a la ulceración central, localizados en la superficie posterior de las piernas, generalmente en mujeres. Se asocia a enfermedad pulmonar $\operatorname{activa}^{8,20}$. 
A la histología se observan tres de cuatro elementos, paniculitis lobulillar, necrosis del tejido adiposo, vasculitis o granulomas ${ }^{8}$.

Las lesiones se resuelven lentamente, aun en presencia de terapia antituberculosa; pueden dejar hiperpigmentación secundaria o una cicatriz atrófica ${ }^{1,20}$.

Liquen escrofulosorum. Se trata de múltiples pápulas pequeñas liquenoides, color piel o rojo pálido, foliculares o parafoliculares, asintomáticas y ubicadas, casi siempre, en el tronco. Ocasionalmente pueden evolucionar a placas discoides. Afecta casi siempre a niños, se asocia a enfermedad pulmonar o linfática. Se encuentra frecuentemente el antecedente de vacunación anti TBC ${ }^{21}$.

\section{Tratamiento}

La TBC cutánea corresponde a un tipo de TBC extrapulmonar, por lo que su tratamiento se basa en el clásico esquema diario por dos meses y bisemanal por cuatro meses (Tabla 2) 4,22. $^{4}$.

Hay que tener en consideración para el tratamiento el tipo de compromiso cutáneo, la etapa de la enfermedad, el nivel de inmunidad y gravedad del paciente ${ }^{4}$.

De acuerdo a las pautas nacionales de tratamiento anti-TBC, se puede iniciar un esquema diario en base a tres fármacos: HIN, RFP y PZ, para luego de dos meses continuar con un esquema bisemanal de HIN y RFP por cuatro meses adicionales ${ }^{4,22}$.

Los fármacos de segunda línea son necesarios para el tratamiento en caso de resistencia, dentro de las que se encuentran kanamicina, amikacina, capreomicina, etionamida y cicloserina; también se pueden usar quinolonas como ofloxacina, levofloxacina, gatifloxacina y moxifloxacina ${ }^{4,23}$.

\section{Discusión}

Nos enfrentaremos con mayor frecuencia a casos de TBC, debido al mencionado aumento de población inmunocomprometida. Es así como también deberían aumentar sus presentaciones menos frecuentes, como es el caso de la TBC cutánea. En pacientes con inmunosupresión, puede ser más de uno el agente causal de las lesiones cutáneas y no es siempre fácil de realizar el estudio etiológico.

En el caso de la primera paciente, se trata de una persona inmunocomprometida por uso de corticoesteroides. Esto aumenta la probabilidad de presentar TBC cutánea, ya que puede facilitar la diseminación a la piel, ya sea hematógena, linfática o directa desde otro foco no cutáneo. Por otro lado, lesiones debidas a re-inoculación del bacilo, también son frecuentes en pacientes con TBC pulmonar, debido al contacto con las manos al toser ${ }^{18}$.
Tabla 2. Tratamiento tuberculosis pulmonar o extra-pulmonar ${ }^{4,22,23}$

\begin{tabular}{lcc} 
& \multicolumn{2}{c}{ Fármacos antituberculosos de primera línea } \\
& Fase diaria (50 dosis) $\mathbf{2}$ meses & Fase bisemanal (32 dosis) $\mathbf{4}$ meses \\
Isoniacida & $5 \mathrm{mg} / \mathrm{kg}-300 \mathrm{mg}$ & $15 \mathrm{mg} / \mathrm{kg}-800 \mathrm{mg}$ \\
Rifampicina & $10 \mathrm{mg} / \mathrm{kg}-600 \mathrm{mg}$ & $10 \mathrm{mg} / \mathrm{kg}-\mathbf{6 0 0} \mathrm{mg}$ \\
Pirazinamida & $25 \mathrm{mg} / \mathrm{kg}-1500 \mathrm{mg}$ & - \\
Etambutol & $20 \mathrm{mg} / \mathrm{kg}-1200 \mathrm{mg}$ \\
& Fármacos antituberculosos de segunda línea \\
Kanamicina & Cicloserina \\
Etionamida & Ácido para-aminosalicílico \\
Moxifloxacina & Gatifloxacina \\
\hline
\end{tabular}

En la segunda paciente no se evidenció una causa de inmunosupresión de base. En este caso tampoco fue posible aislar M. tuberculosis; sin embargo, la expresión clínica era sugerente y la biopsia apoyó el diagnóstico con lo que se inició el tratamiento, observándose resolución de las lesiones. Ante el bajo rendimiento de los exámenes microbiológicos y el largo tiempo de incubación de los cultivos, hasta 60 días, si el cuadro clínico es sugerente pero no se puede confirmar el diagnóstico con los exámenes complementarios, la respuesta al tratamiento empírico con terapia anti TBC es un criterio diagnóstico ${ }^{7,18}$. La duración de esta prueba terapéutica debe ser de aproximadamente cinco semanas y, en caso de experimentar una buena evolución, se completa el tratamiento ${ }^{24}$. La emergencia de bacilos resistentes a múltiples de estos fármacos y la toxicidad de la terapia complica este abordaje.

En el enfrentamiento diagnóstico de casos con sospecha de TBC, siempre se debe incluir el estudio microbiológico con baciloscopia y cultivo de Koch. Hay que considerar que la sensibilidad de los exámenes varía de acuerdo al tipo de TBC cutánea, siendo mayor para las formas multibacilares. Gran utilidad puede tener la RPC en casos en que se demuestren bacilos ácido-alcohol resistentes en la biopsia, para diferenciar entre TBC e infección con micobacterias atípicas en pacientes inmunocomprometidos, teniendo en cuenta que los cultivos pueden ser negativos o tardar demasiado ${ }^{25,26}$.

Los casos presentados confirman la importancia de mantener una vigilancia activa sobre lesiones cutáneas que puedan ser secundarias a infección por M. tuberculosis ante los que se debe realizar los estudios correspondientes para su apropiado y oportuno diagnóstico.

\section{Resumen}

La tuberculosis (TBC) cutánea es una enfermedad infecciosa crónica causada por Mycobacterium tubercu- 
losis. Es una patología poco frecuente (1\% de todos los casos de TBC); no obstante, ha aumentado debido a la epidemia del virus de la inmunodeficiencia humana y la presencia creciente de la inmunosupresión farmacológica. Presenta una gran variedad de formas clínicas que dependen de cómo llega el bacilo a la piel y del estado inmunológico del individuo. A continuación, presentamos dos casos clínicos de TBC cutánea vistos en el Hospital Clínico de la Pontificia Universidad Católica de Chile y una revisión del tema.

\section{Referencias}

1.- Barbagallo J, Tager P, Ingleton R, Hirsch R J, Weinberg J M. Cutaneous tuberculosis: diagnosis and treatment. Am J Clin Dermatol 2002; 3 (5): 319-28.

2.- Zúñiga M M P, Riquelme C. Reflections about tuberculosis in Chile. Rev Chil Enf Respir 2007; 23: 59-66.

3.- World Health Organization. Global Tuberculosis Database. Consultado en http://apps.who.int/ globalatlas/dataQuery/default.asp el 25 de junio de 2009.

4.- Handog E B, Gabriel T G, Pineda R T. Management of cutaneous tuberculosis. Dermatol Ther 2008; 21 (3): 154-61.

5.- Suárez J M, Herrera L, Bautista F J, Dávila J. Skin abscess in a one-year-old infant. Enferm Infecc Microbiol Clin 2004; 22 (6): 361-2.

6.- Mataix J, Botella R, Herrero A, Lucas A. Tuberculous primary complex of the skin. Int J Dermatol 2008; 47 (5): 479-81.

7.- Saritha M, Parveen B A, Anandan V, Priyavathani M R, Tharini K G. Atypical forms of lupus vulgaris - a case series. Int J Dermatol 2009; 48 (2): 150-3.

8.- $\quad$ Bravo F G, Gotuzzo E. Cutaneous tuberculosis. Clin Dermatol 2007; 25 (2): 173-80.

9.- Pérez B, Pifarre R, de Vera CV, García J M, Baradad M, Vila M, et al. Cutaneous tuberculosis caused by Mycobacterium tuberculosis, an uncommon pathology. An Med Interna 2006; 23 (11): 560-1.

10.- Tanaka R, Matsuura H, Kobashi Y, Fujimoto W. Clinical utility of an interferon-gamma- based assay for mycobacterial detection in papulonecrotic tuberculid. Br J Dermatol 2007; 156 (1): 169-71.

11.- Centers for Disease Control and Prevention. Guidelines for the investigation of contacts of persons with infectious tuberculosis and Guidelines for using the QuantiFERON®TB Gold test for detecting Mycobacterium tuberculosis infection, United States. Consultado en http://www.cdc.gov/mmwr/pdf/ rr/rr5415.pdf el 19 de julio de 2009.

12.- Villarroel L, Rabagliati R, Balcells ME, Karzulovic L, Pérez C. Prevalence of tuberculosis and its impact on mortality among HIV infected patients in Chile. Rev Med Chile 2008; 136 (5): 578-86.

13.- Kazandjieva J, Tsankov N. Tattoos: dermatological complications. Clin Dermatol 2007; 25 (4): 375-82.

14.- Sehgal V N, Wagh S A. Cutaneous tuberculosis. Current concepts. Int J Dermatol 1990; 29 (4): 237-52.

15.- Semaan R, Traboulsi R, Kanj S. Primary Mycobacterium tuberculosis complex cutaneous infection: report of two cases and literature review. Int J Infect Dis 2008; 12 (5): 472-7.

16.- Zeegelaar J E, Faber W R. Imported tropical infectious ulcers in travelers. Am J Clin Dermatol 2008; 9 (4): 219-32.

17.- Sehgal VN, Sehgal R, Bajaj P, Sriviastava G, Bhattacharya $S$. Tuberculosis verrucosa cutis (TBVC). J Eur Acad Dermatol Venereol 2000; 14 (4): 319-21.

18.- Pizzariello G, Fernández P, D’Atri G, Novac V,
Uranga A. Espectro clínico de tuberculosis cutánea. Rev Argent Dermatol 2008; 89: 177-87.

19.- Seyahi N, Apaydin S, Kahveci A, Mert A, Sariyar M, Erek E. Cellulitis as a manifestation of miliary tuberculosis in a renal transplant recipient. Transpl Infect Dis 2005;7 (2): 80-5.

20.- Mascaro J, Baselga E. Erythema induratum of Bazin. Dermatol Clin 2008; 26 (4): 439-45.

21.- Sehgal V N. Lichen scrofulosorum: current status. Int J Dermatol 2005; 44 (6): 521-3.

22.- Minsal. Programa Nacional de Control de la Tuberculosis 2005. Manual de Organización y Normas Técnicas. Consultado en www.redsalud. gov.cl/archivos/TUBERCULOSIS.pdf el 30 de junio de 2009.

23.- Centers for Disease Control and Prevention. Treatment of Tuberculosis. MMWR Morbid Mortal Wkly Rep 2003; 52 (No. RR-11).

24.- Ramam M, Tejasvi T, Manchanda Y, Sharma S, Mittal R. What is the appropriate duration of a therapeutic trial in cutaneous tuberculosis? Further observations. Indian J Dermatol Venereol Leprol 2007; 73 (4): 243-6.

25.- Tan S H, Tan B H, Goh C L, Tan K C, Tan M F, $\mathrm{Ng}$ W C, et al. Detection of Mycobacterium tuberculosis DNA using polymerase chain reaction in cutaneous tuberculosis and tuberculids. Int J Dermatol 1999; 38 (2): 122-7.

26.- Tan S H, Tan H H, Sun Y J, Goh C L. Clinical utility of polymerase chain reaction in the detection of Mycobacterium tuberculosis in different types of cutaneous tuberculosis and tuberculids. Ann Acad Med Singapore 2001; 30 (1): 3-10. 